\title{
2 Impact of Nonlinearities on Fiber Optic
}

3 Communications

\author{
Mário Ferreira \\ I3N-Institute of Nanostructures, Nanomodelling and Nanofabrication, Department of Physics, University of \\ Aveiro 3810-193 Aveiro, Portugal \\ * Correspondence: mfernando@ua.pt; Tel.: +351-234370279
}

\begin{abstract}
A number of third order nonlinear processes can occur in single-mode fibres and an understanding of such phenomena is almost a prerequisite for actual lightwave-system designers. In this paper we review the main limitations imposed by several nonlinear effects, namely the selfand cross-phase modulation, four-wave mixing, stimulated Raman scattering and stimulated Brillouin scattering, on the performance of optical fiber communication systems.
\end{abstract}

Keywords: Optical fibers: optical fiber communications; nonlinear fiber optics

\section{Introduction}

Glass fibres for optical communications are made of fused silica, an amorphous material, to which dopant materials of various kinds can be added to produce changes in refractive index. A number of third order nonlinear processes can occur; these can grow to appreciable magnitudes over the long lengths available in fibres, even though the nonlinear coefficients in the materials are relatively small. The effects are particularly important in single-mode fibres, in which the small mode field dimensions result in substantially high light intensities with relatively modest input powers.

Fiber nonlinearities fall into two general categories [1]. The first category of nonlinearities arises from modulation of the refractive index of silica by intensity changes in the signal (Kerr effect). This gives rise to nonlinearities such as self-phase modulation (SPM), whereby an optical signal alters its own phase; cross-phase modulation (XPM), where one signal affects the phases of all others optical signals and vice-versa; and four-wave mixing (FWM), whereby signals with different frequencies interact to produce mixing sidebands. The second category of nonlinearities corresponds to stimulated scattering processes, such as stimulated Brillouin scattering (SBS) and stimulated Raman scattering (SRS), which are interactions between optical signals and acoustic or molecular vibrations in the fiber.

Fiber nonlinearities have different influences on the communication systems. The SPM, for instance, leads to a change in the dispersion behaviour in high-bit-rate transmission systems; the XPM, SRS, and SBS determine a decrease of the signal to noise ratio; the SRS and FWM will increase the crosstalk between different WDM channels [1]. On the other hand, the same nonlinear effects offer a variety of possibilities for ultrafast all-optical switching, amplification and regeneration [1,2]. The FWM, SRS, ans SBS, for instance, are able to amplify optical signals in spectral ranges that can never be reached by erbium-doped fiber amplifiers. The FWM offers the possibility for a pure optical wavelength conversion and the realization of nonlinear optical phase conjugation, that can compensate completely the distortions of the optical pulses. Optical solitons offer the possibility of transmitting optical pulses over extremely large distances without distortion $[3,4]$

In this paper we review the main limitations to the performance of optical fiber communication systems arising from fiber nonlinearities. In Section 2 we review the limitations imposed by SPM, XPM, and FWM effects, whereas in Section 3 we consider those limitations due to SRS and SBS effects. Section 4 summarizes the main conclusions. 


\section{Kerr Effect}

Nonlinear effects are attributed to the dependence of the susceptibility on the electric field, which becomes important at high field strengths. As a result, the total polarization vector $\mathbf{P}$ can be written in the frequency domain as a power series expansion in the electric field vector [5]:

$$
\mathbf{P}(\mathrm{r}, \omega)=\varepsilon_{0}\left|\chi^{(1)} \cdot \mathbf{E}+\chi^{(2)}: \mathbf{E E}+\chi^{(3)}: \mathbf{E E E}+\ldots\right|=\mathbf{P}_{L}(\mathrm{r}, \omega)+\mathbf{P}_{N L}(\mathrm{r}, \omega)
$$

where $\chi^{(j)}(j=1,2, \ldots)$ is the $j$ th order susceptibility. To account for the light polarization effects, $\chi^{(j)}$ is a tensor of rank $j+1$. The linear susceptibility $\chi^{(1)}$ determines the linear part of the polarization $\mathbf{P}_{L}$. On the other hand, terms of second and higher order in Eq. (1) determine the nonlinear polarization $\mathbf{P}_{N L}$. Since $\mathrm{SiO}_{2}$ is a symmetric molecule, the second-order susceptibility $\chi^{(2)}$ vanishes for silica glasses. As a consequence, virtually all nonlinear effects in optical fibers are determined by the third order susceptibility $\chi^{(3)}$. In time domain, the form of the expansion is identical to Eq. (1) if the nonlinear response is assumed to be instantaneous.

The presence of $\chi^{(3)}$ implies that the refractive index depends on the field intensity, $I$, in the form

$$
n=\sqrt{1+\chi^{(1)}+\frac{3}{2} \frac{\chi^{(3)}}{c \varepsilon_{0} n_{0}} I} \approx n_{0}+n_{2} I
$$

where $n_{0}=\sqrt{1+\chi^{(1)}}$ is the linear refractive index and $n_{2}=3 \chi^{(3)} /\left(4 c \varepsilon_{0} n_{0}^{2}\right)$ is the refractive index nonlinear coefficient, also known as the Kerr coefficient.

In the case of silica fibers, we have $n_{0} \approx 1.46$ and $n_{2} \approx 3.2 \times 10^{-20} \mathrm{~m}^{2} / W$. Considering a single-mode fiber with an effective mode area $A_{\text {eff }}=50 \mu \mathrm{m}^{2}$ carrying a power $P=100 \mathrm{~mW}$, the nonlinear part of the refractive index is $n_{2} I=n_{2}\left(P / A_{\text {eff }}\right) \approx 6.4 \times 10^{-11}$. In spite of this very small value, the effects of the nonlinear component of the refractive index become significant due to very long interaction lengths provided by the optical fibers.

The Kerr nonlinearity gives rise to different effects, depending on the shape of the field injected into the fiber. In the following, the main effects due to Kerr nonlinearity and the limitations imposed by them on lightwave communication systems will be reviewed. 


\subsection{Self-Phase Modulation}

The change in refractive index due to the Kerr effect determines a corresponding change in the propagation constant. As a consequence, the phase of a signal propagating through the fiber varies with distance according to the equation:

$$
\phi=n_{0} k_{0} z+\gamma P(t) z
$$

where $\gamma=n_{2} k_{0} / A_{\text {eff }}$. The first term in Eq. (3) represents the linear phase shift due to signal

propagation; the second term represents the nonlinear phase shift. When the incident wave is a pulse with a power variation given by $P(t)$, the output pulse is chirped. This phenomenon is called selfphase modulation (SPM), since the power variation within the pulse leads to its own phase modulation. In the leading edge of the pulse, where $d P / d t>0$, the instantaneous frequency is downshifted from the central frequency, whereas in the trailing edge, where $d P / d t<0$, the instantaneous frequency is upshifted. The chirping due to nonlinearity leads to increased spectral broadening.

The maximum phase shift due to SPM is given by

$$
\phi_{N L}=\gamma P_{0} L_{e f f}
$$

where $P_{0}$ is the peak power of the pulse and

$$
L_{e f f}=N \frac{1-e^{-\alpha L_{A}}}{\alpha}
$$

is the effective length of the transmission link, $\alpha$ being the fiber attenuation coefficient, $L_{A}$ the spacing between consecutive amplifiers, and $N=L / L_{A}$ the number of sections constituting the transmission link. When $\alpha L » 1$ the effective length approaches a limiting value, given by:

$$
L_{\text {eff }}^{\max }=\frac{N}{\alpha}
$$

The phase shift given by Eq, (4) becomes significant $(\sim \pi / 2)$ when the power times the net effective length of the system reaches $1 \mathrm{~W} . \mathrm{km}$ or $1 \mathrm{~mW} . \mathrm{Mm}$. The first set of units is appropriate for repeaterless systems and the second for long amplified systems. In the first case the effects of SPM are of little concern, since other nonlinear effects, namely stimulated Brillouin scattering, limit themselfs power levels to below $10 \mathrm{~mW}[6,7]$. In the second case, however, SPM can be a major limiting factor, since its effects accumulate over the entire link and the maximum phase shift increases linearly with the number of amplifiers, $N$. Considering $L_{\text {eff }} \approx N / \alpha$ and using typical values, we find that the peak power is limited to below $3 \mathrm{~mW}$ for links with only 10 amplifiers.

The impact of the SPM effects on the transmission system depends on the modulation format of the carrier. For example, in the case of phase binary shift keying (PSK) systems the information lies in the carrier phase, which changes between $+\pi / 2$ and $-\pi / 2$. Phase noise leads to a reduction of the signal to noise ratio (SNR), which can be significant if semiconductor lasers are directly phasemodulated, due to their strong intensity fluctuations.

In the presence of dispersion, the spectral broadening due to SPM determines two situations qualitatively different. In the normal dispersion region (wavelength shorter than the zero dispersion wavelength) the chirping due to dispersion corresponds to a downshift of the leading edge and to an upshift of the trailing edge of the pulse, which is a similar effect as that due to SPM. Thus, in this regime the chirping due to dispersion and SPM act in the same direction and lead to a stronger temporal broadening of the pulse than the dispersion alone, thus determining a more significant reduction of the system capacity.

If the pulse, spectrally-broadened by SPM, is transmitted in the anomalous dispersion regime, the red-shifted leading edge travels more slowly, and moves toward the pulse center. Similarly, the trailing edge of the pulse, which has been blue-shifted, travels more quickly, and also moves toward 
the center of the pulse. Therefore, GVD and SPM act in different directions, resulting in a compression of the pulse.

In the range of anomalous dispersion, nonlinearity and dispersion induced chirpings can partially or even completely cancel each other. When this cancellation is total, the pulse neither broadens in time nor in its spectrum and such pulse is called a fundamental soliton.

\subsection{Cross-Phase Modulation}

When two or more signals having different carrier frequencies are transmitted simultaneously inside an optical fiber, the nonlinear phase evolution of the signal at frequency $\omega_{i}$ depends also on the power of the other signals. This nonlinear phenomenon is known as cross-phase modulation (XPM) and it is due to the intensity dependence of the refractive index in Eq. (5). The nonlinear phase shift of the signal at $\omega_{j}$ becomes:

$$
\phi_{j}=\gamma_{j} L_{e f f}\left[P_{j}+2 \sum_{m \neq j}^{M} P_{m}\right]
$$

where $P_{m}$ is the power of the signal at $\omega_{m}$. The first term in the square brackets represents the contribution of SPM, while the second term is the contribution from the XPM. The factor 2 in Eq. (7) indicates that XPM is twice as effective as SPM for the same amount of power.

The effect of XPM is different in amplitude- and in phase-modulated systems. In the last case, since the power in each channel is the same for all bits, the main limitation results from arbitrary phase fluctuations, which lead directly to a deterioration of the signal-to-noise ratio. Such phase fluctuations can be induced via the XPM by intensity variations, as happen if semiconductor lasers are directly phase-modulated.

In the case of amplitude-modulated direct detection systems, the XPM has no effect on the system performance if the dispersion is neglected. Actually, since the phase alteration due to XPM is associated with a frequency alteration, the dispersion determines an additional temporal broadening or compression of the spectral broadened pulses, which affects the system performance.

The impact of XPM is particularly significant in the case of amplitude-modulated coherent communication system, employing a phase-sensitive detection scheme. In fact, the phase in a given channel depends on the bit pattern of neighboring channels. In the worst case, in which all channels have " 1 " bits in their time slots, the XPM-induced phase shift is maximum. Assuming a repeaterless system such that the power $P$ in each channel is the same, this phase shift is given by

$$
\phi_{\max }=\frac{\gamma}{\alpha}(2 M-1) P
$$

where it was assumed that $\alpha L » 1$. Considering a maximum tolerable phase shift $\phi_{\max }=0.1$, the power in each channel is limited to

$$
P<\frac{\alpha}{10 \gamma(2 M-1)}
$$

For typical values of $\alpha$ and $\gamma, P$ should be below $1 \mathrm{~mW}$ even for five channels.

The impact of XPM would be negligible in frequency- or phase-modulated coherent systems if the channel powers were really constant in time. However, this is not the case in practice, since the intensity noise of the transmitters or the ASE noise added by the optical amplifiers cause fluctuations of the channel powers. XPM converts such fluctuations into phase fluctuations, which degrade the performance of the coherent receiver.

The XPM effect determines a mutual influence between two pulses only if they overlap at some extent. However, in the presence of finite dispersion, the two pulses with different wavelengths will move with different velocities and thus will walk off from each other. If the pulses enter the fiber 
164

165

166

167

168

169

170

171

172

173

\section{4}

175

176

177

178

179

180

181

182

183

184

185

186

187

188

189

190

191

192

separately, walk through each other and again become separated, it is said that they experience a complete collision. In a lossless fiber, such collision is perfectly symmetric and no residual phase shift remains, since the pulses would have interacted equally with both the leading and the trailing edge of the other pulse. However, in case the pulses enter the fiber together the result is a partial collision, since each pulse will see only the trailing or the leading edge of the other pulse, which will lead to chirping. Moreover, in the case of a periodically amplified system, power variations also make complete collisions asymmetric, resulting in a net frequency shift that depends on the wavelength difference between the interacting pulses. Such frequency shifts lead to timing jitter in multichannel systems, since their magnitude depends on the bit pattern as well as on channel wavelengths. The combination of amplitude and timing jitter degrades significantly the system performance [8].

\subsection{Four-Wave Mixing}

Four-wave mixing (FWM) is a parametric interaction among waves satisfying a given phase relationship called phase matching. Different phenomena may be originated by FWM process depending on the relation among interaction frequencies. If three optical fields with carrier frequencies $\omega_{i}(i=1,2,3)$ copropagate inside the fiber simultaneously, it appears that the third-order polarization vector has several components: three components have the frequencies of the input fields, the others have an angular frequency $\omega_{4}$ given by

$$
\omega_{4}=\omega_{1} \pm \omega_{2} \pm \omega_{3}
$$

If no field is present in the fiber at the frequency $\omega_{4}$, a new field component is created at this frequency. If a field at the frequency $\omega_{4}$ is already present in the fiber, it will be affected by the nonlinear interaction between the fields at $\omega_{i}$, which causes crosstalk in multichannel communication systems.

The phase-mismatch among all four waves is given by

$$
\Delta \beta=\beta\left(\omega_{1}\right)+\beta\left(\omega_{2}\right)-\beta\left(\omega_{3}\right)-\beta\left(\omega_{4}\right)
$$

where $\beta(\omega)$ is the propagation constant for an optical field with frequency $\omega$. Assuming that the frequencies are closely and equally spaced (i.e., $\omega_{1}=\omega_{2}-\Delta \omega, \omega_{3}=\omega_{2}-2 \Delta \omega, \omega_{1}=\omega_{2}-\Delta \omega$ ) and making a Taylor series expansion of all $\beta$ s about the frequency $\omega_{2}$, we get

$$
\Delta \beta=2 \beta_{2}(\Delta \omega)^{2}
$$

where $\beta_{2}=\partial^{2} \beta / \partial \omega^{2}$ is the group velocity dispersion (GVD). When $\beta_{2}=0$ we have a perfect phase matching and thus an efficient FWM. This situation is desirable for applications such as alloptical signal processing, wavelength conversion, pulse compression, etc. [1,2]. However, in WDM systems FWM causes a transfer of power from each channel to its neighbors. Such a power transfer not only results in the power loss for the channel but also induces interchannel crosstalk that degrades the system performance severely.

In the case of WDM systems with equal channel spacing, the degradation due to FWM is particularly severe, since in this case most new frequencies coincide with the original channel frequencies. The interference between the original and the new generated waves depends on the bit pattern and leads to significant fluctuations in the detected signal at the receiver, thus increasing the BER in the system. Note that in systems with channels equally spaced in wavelength the frequency spacing will not be uniform. However, the unequal frequency spacing in this case is not sufficient to prevent interference. The difference in frequency spacing, and hence the offset of mixing product from the channel must be at least twice the bit rate to avoid interference [9]. To prevent the coincidence of the mixing products with any channel, the difference between any two channel frequencies must be unique [9]. Such objective can be achieved with a computer search. 
In the case of WDM systems with unequal channel spacing, crosstalk due to FWM is suppressed, since the new frequencies fall in between the existing channel frequencies and only add to overall noise. The use of unequal channel spacings to reduce the FWM-induced degradation was shown to be effective in a 1999 experiment, in which 22 channels, each operating at $10 \mathrm{~Gb} / \mathrm{s}$, were transmitted over $320 \mathrm{~km}$ of dispersion-shifted fiber with 80-km amplifier spacing [10].

Even for a non-zero value of dispersion, the FWM process can be resonantly enhanced for certain values of channel spacing due to the contribution of SPM and XPM [11]. In fact, both these effects can produce phase matching when the GVD is in the anomalous regime [1]. The resonance enhancement of FWM occurs if the frequency of the gain peak of modulation instability nearly coincides with the channel spacing in a WDM system. Such channel spacing is approximately given by [1]:

$$
\Delta f_{c h}=\frac{1}{2 \pi}\left(\frac{2 \gamma P_{c h}}{\left|\beta_{2}\right|}\right)^{1 / 2}
$$

Considering the values $P_{c h}=5 \mathrm{~mW}, \beta_{2}=-0.1 \mathrm{ps}^{2} / \mathrm{km}$, and $\gamma=2 \mathrm{~W}^{-1} / \mathrm{km}$, we obtain a channel spacing $\Delta f_{c h} \approx 70 \mathrm{GHz}$, which is within the range usually considered in modern WDM systems.

In spite of the advantages of using fibers with high local dispersion to reduce the FWM efficiency, it is also very important to have a small dispersion of the fiber span in the case of high bit rate communication systems. A solution for the above dilemma is provided by the technique of dispersion-management. In this case, fibers with normal and anomalous dispersion are combined to form a periodic dispersion map, such that the local GVD is high but its average value is kept low. Due to its simplicity of implementation, the dispersion-management technique became quite common since 1996 to control the FWM-induced limitations in WDM systems [12].

\section{Stimulated Light Scattering}

Stimulated scattering processes, such as stimulated Raman scattering (SRS) and stimulated Brillouin scattering (SBS), correspond to interactions between optical signals and acoustic or molecular vibrations in the fiber, respectively. Both these processes are inelastic, since they can be understood as scattering of a photon to a lower energy photon, such that the energy difference appears in the form of a phonon: an optical phonon in Raman scattering and an acoustic phonon in Brillouin scattering

\subsection{Stimulated Raman Scatering}

Stimulated Raman scattering occurs as a consequence of the coherent interaction between the optical fields of the incident wave (also called the pump wave) and of the new frequency-shifted wave (also called the Stokes wave). This interaction originates a driving force that excites the molecular resonances. In a quantum mechanical description, one has simultaneously the absorption of a photon from the pump beam at frequency $\omega_{p}$ and the emission of a photon at the Stokes frequency $\omega_{s}$. The difference in energy is taken up by a high energy phonon (molecular vibration) at frequency $\omega_{v}$.

The pump wave intensity $\left(I_{p}\right)$ and the Stokes wave intensity $\left(I_{S}\right)$ satisfy the following equations [1]:

$$
\frac{d I_{S}}{d z}=g_{R} I_{S} I_{P}-\alpha I_{S}
$$




$$
\frac{d I_{P}}{d z}=-\frac{\omega_{P}}{\omega_{S}} g_{R} I_{S} I_{P}-\alpha I_{P}
$$

where $\alpha$ takes into account the fiber losses and $g_{R}$ is the Raman gain coefficient. The most significant feature of the Raman gain in silica fibres is that $g_{R}$ extends over a large frequency range (up to $40 \mathrm{THz}$ ) with a broad dominant peak near $13 \mathrm{THz}$. This behaviour is due to the amorphous nature of silica glass, whose molecular vibrational energy levels merge together to form a band. The peak value of the Raman gain coefficient for silica fibres is $9.4 \times 10^{-14} \mathrm{~m} . \mathrm{W}^{-1}$ for a pumping wavelength $\lambda_{p}=1.0 \mu \mathrm{m}$ and varies as $\lambda_{p}^{-1}$ [13].

When the input Stokes wave intensity is weak, such that $I_{S 0} \ll I_{P 0}$, the evolution of the Stokes wave intensity is given from Eq.s (14) and (15) approximately by:

$$
I_{S}(z) \approx I_{S 0} \exp \left\{\frac{g_{R} I_{P 0}[1-\exp (-\alpha z]}{\alpha}-\alpha z\right\}
$$

In the absence of an input signal $I_{S 0}$, the Stokes wave arises from spontaneous Raman scattering along the fiber. The threshold for stimulated Raman scattering is defined as the input pump power at which the output powers for pump and Stokes wave become equal. In long polarizationmaintaining fibers, such that $L_{\text {eff }} \approx 22 \mathrm{~km}$, and considering an effective core area of $A_{\text {eff }}=50 \mu \mathrm{m}^{2}$, the threshold for the stimulated Raman scattering is $P_{P 0}^{t h} \approx 600 \mathrm{~mW}$ at $\lambda_{P}=1.55 \mu \mathrm{m}$. However, in standard single-mode fibers with similar characteristics, the threshold would be $P_{P 0}^{t h} \approx 1.2 \mathrm{~W}$.

Because SRS has a relatively high threshold, it is not of concern for single-channel systems. However, in WDM systems SRS can cause crosstalk between channels signals whose wavelength separation falls within the Raman gain curve. Specifically, the long-wavelength signals are amplified by the short-wavelength signals, leading to power penalties for the latter signals. The shortestwavelength signal is the most depleted, since it acts as a pump for all other channels. The Ramaninduced power transfer between two channels depends on the bit pattern, which leads to power fluctuations and determines additional receiver noise. The magnitude of these deleterious effects depends on several parameters, like the number of channels, their frequency spacing, and the power in each of them.

If dispersion is neglected and considering the worst case of "one" bits being simultaneously transmitted on all $N$ channels of a WDM system, spaced by $\Delta f_{c h}$ and each of them carrying a power $P_{c h}$, it can be shown that the product of total power $\left(N P_{c h}\right)$ and total bandwidth ( (N-1) $\left.\Delta f_{c h}\right)$ must be smaller than $500 \mathrm{GHz}-\mathrm{W}$ to guarantee a penalty for the shortest wavelength channel lower than $1 \mathrm{~dB}$ [14].

In WDM systems that contain no optical amplifiers, the SRS leads to a power reduction of the short wavelength channels and, therefore, a degradation of the SNR. However, in long haul transmission systems, a number of optical amplifiers is generally used. Besides providing the desired amplification of the signal, such optical amplifiers add also noise. Since noise is added periodically over the entire length of a system, it experiences less Raman loss than the signal. For small degradations, the fractional depletion of the noise is half the fractional depletion of the signal. Therefore, the SRS reduces the SNR and the capacity in amplified systems.

Raman crosstalk can be suppressed by reducing the channell power, but such approach may not be practical in some circumstances. Another possibility is to use the technique of mid-span spectral 
inversion [15]. This technique leads to an inversion of the whole WDM spectrum in the middle of the transmission link. Hence, channels with higher wavelengths would become short-wavelength channels and vice-versa. As a result, the direction of Raman-induced power transfer will be reversed in the second half of the fiber span and a balance of the channel powers will be achieved at the end of the fiber link. Spectral inversion can be realized inside a fiber through phase conjugation provided by the FWM effect.

\subsection{Stimulated Brillouin Scatering}

The process of stimulated Brillouin scattering (SBS) can be described as a classical three-wave interaction involving the incident (pump) wave of frequency $\omega_{p}$, the Stokes wave of frequency $\omega_{s}$ and an acoustic wave of frequency $\omega_{a}$. The pump creates a pressure wave in the medium owing to electrostriction, which in turn causes a periodic modulation of the refractive index. Physically, each pump photon in the SBS process gives up its energy to create simultaneously a Stokes photon and an acoustic phonon.

The three waves involved in the SBS process must conserve both the energy and the momentum. The energy conservation requires that $\omega_{p}-\omega_{s}=2 \pi f_{a}$, where $f_{a}$ is the linear frequency of the acoustic wave, which is about $11.1 \mathrm{GHz}$ in standard fibers. The momentum conservation requires that the wave vectors of the three waves satisfy $\mathbf{k}_{a}=\mathbf{k}_{p}-\mathbf{k}_{s}$. In a single-mode fiber, optical waves can propagate only along the direction of the fiber axis. Since the acoustic wave velocity $v_{a} \approx 5.96$ $\mathrm{km} / \mathrm{s}$ is by far smaller than the light velocity, $\left|\mathbf{k}_{a}\right|=2 \pi f_{a} / v_{a}>\left|\mathbf{k}_{p}\right| \approx\left|\mathbf{k}_{s}\right|$. In this case the momentum conservation has the important consequence that Brillouin effect occurs only if the Stokes and the pump waves propagate in opposite directions.

In the SBS process, the Stokes mode intensity, $I_{s}$, and the pump intensity , $I_{p}$, satisfy equations similar to (14) and (15), in which the Raman gain coefficient, $g_{R}$, is replaced by the Brillouin gain coefficient, $g_{B}$. This coefficient is estimated to be about $2.5 \times 10^{-11} \mathrm{~m} . \mathrm{W}^{-1}$ for typical fibers, a value which is two orders of magnitude larger than the Raman gain coefficient at $\lambda_{p}=1.55 \mu \mathrm{m}$.

SBS can affect the performance of a transmission system by several ways. First, the threshold of the SBS process determines the maximum power which can be launched into the system. Such maximum power can be of the order of some few $\mathrm{mW}$. This fact limits the maximum SNR and the transmission distance which can be reached without amplification. Once the SBS threshold is surpassed, as a consequence of the power transfer to the Stokes wave, the pump signal is depleted, which determines again a degradation of the SNR and leads to an increase of the BER. Moreover, the backward propagating Stokes wave can destabilize and even destroy the signal transmitter if no optical isolator is appropriately inserted in the system.

In actual transmission systems optical amplifiers are periodically inserted to compensate for the fiber losses. Each amplifier includes generally an optical isolator, which avoids the passage and successive growth of the backward propagating Stokes wave. In spite of this action, SBS between consecutive amplifiers still can degrade the system performance if the signal power is above the threshold.

Another main detrimental effect of SBS is related with the interchannel crosstalk in WDM systems. Such crosstalk occurs only if the fiber link supports the propagation of channels in opposite direction and if the channel spacing between two counterpropating channels is approximately equal to the Brillouin shift $(\sim 11 \mathrm{GHz})$. If both these conditions are fulfilled, the channel with the Stokes frequency is amplified at the expense of the channel with the pump frequency. In fact, impairments 
resulting from SBS-induced crosstalk can be observed in bidirectional transmission systems at power levels far below the SBS threshold [16]. However, this kind of crosstalk can be easily suppressed with a slight change of the channel spacing.

Much attention has been paid to estimating the SBS limitations in practical fiber transmission systems. SBS is very sensitive to signal modulation because the origin of SBS involves a process which is not instantaneous on the time scale of the information rate. The narrow Brillouin linewidth is a consequence of the long lifetimes of the acoustic phonons involved in light scattering. In general, high modulation rates produce broad optical spectra, which will determine a reduction of the Brillouin gain.

Concerning the coherent transmission systems, the SBS threshold depends on whether the amplitude, phase, or frequency of the optical carrier is modulated for information coding. Assuming a fixed bit pattern and that the fundamental modulation frequency for ASK and PSK, as well as that the difference between the two frequencies of the FSK is much higher than the bandwidth of the Brillouin gain, it can be shown that the powers of the distinct spectral components of pump and Stokes wave satisfy a pair of coupled equations similar to Eq. (14) and (15) [17]. In these circumstances, the different frequency components of the modulated wave will not influence each other. For WDM systems, SBS will not occur if each frequency in each individual channel remains below threshold. Within the same conditions, it was shown that the threshold for ASK, PSK and FSK systems is 2, 2.5, and 4 times, respectively, that of a CW wave [17].

\section{Conclusions}

In this paper we presented a review of several the nonlinear effects occurring in optical fibers, namely the self- and cross-phase modulation, four-wave mixing, stimulated Raman scattering and stimulated Brillouin scattering. The main limitations imposed by these nonlinear effects on the performance of optical fiber communication systems were also discussed. Besides such limitations, the same effects offer also new possibilities and can find useful applications, namely in the areas of all-optical signal processing, amplification and regeneration.

Acknowledgments:

We acknowledge FCT (Fundação para a Ciência e Tecnologia) for supporting this work through the Project UID/CTM/50025/2013. 
368

369

370

\section{References}

1. Ferreira, M. F., Nonlinear Effects on Optical Fibers, (John Wiley \& Sons, Hoboken, New Jersey, 2011).

2. Agrawal, G. P., Applications of Nonlinear Fiber Optics (Academic Press, San Diego, 2001).

3. Hasegawa, A., (Ed.), New Trends in Optical Soliton Transmission Systems (Kluwer Academic Publishers, AH Dordrecht, The Netherlands, 1998).

4. Ferreira, M. F., Fiber Integrat. Optics, 24, pp. 287 (2005).

5. Butcher, P. N., and Cotter, D. N., The Elements of Nonlinear Optics (Cambridge University Press, Cambridge, UK, 1990).

6. Smith, R. G., Appl. Opt. 11, 2489 (1972).

7. Mao, X. P., Tkach, R. W., Chraplyvy, A. R., Jopson, R. M., and Derosier, R. M., IEEE Photon. Technol. Lett. 4, 66 (1992).

8. Marcuse, D., Chraplyvy, A. R., and Tkach, R. W., J. Lightwave Technol. 12, 885 (1994).

9. Forghieri, F., Tkach, R. W., and Chraplyvy, A. R., IEEE Photon. Technol. Lett. 6, 754 (1994).

10. Suzuki, H., Ohteru, S., and Takachio, N., IEEE Photon. Technol. Lett. 11, 1677 (1999).

11. Grosz, D. F., Mazzali, C., Celaschi, S., Paradisi, A., and Fragnito, H. L., IEEE Photon. Technol. Lett. 11, 379 (1999).

12. Agrawal, G. P., Fiber-Optic Communication Systems (3th ed. Wiley, New York, 2002).

13. Buck, J. A., Fundamental of Optical Fibers, 2nd ed. (Wiley, New York, 2004).

14. Chraplyvy, A. R., J. Lightwave Technol. 8, 1548 (1990).

15. Chraplyvy, A. R., and Tkach, R. W., IEEE Photon. Technol. Lett. 5, 666 (1993).

16. van Deventer, M. O., van der Tol, J. J., and Boot, A. J., IEEE Photon. Technol. Lett. 6, 291 (1994).

17. Aoki, Y., Tajima, K., Mito, I., J. Lightwave Technol. 6, 710 (1988). 